\title{
DETECTION OF SENTINEL LYMPH NODE IN PATIENTS WITH EARLY-STAGE CERVICAL CANCER WITH PATENT BLUE, IN OSECAC.
}

Nieva A, Gomez Cherey F, Cardozo Gutiérrez R, Torres G, Lange J, Levit C, Alessandria S. Section Gynecologic Oncology, OSECAC.

INTRODUCTION: Women with early-stage (stages la1-lb1 FIGO 2014) of cervical cancer (CC), node involvement is one of the most important prognostic factors and guides postoperative adjuvant therapy.

OBJECTIVE: To evaluate the detection percentage of the SLN and its location. Determine whether performing only SLN reduces morbidity.

MATERIAL METHODS: This is retrospective descriptive study. Between Jan/2015, and Sep/2018. Sixty-one women with CC stages la1 to lb1 were operated (epidermoid and adenocarcinoma lineage) in OSECAC by the same surgical team. In 37 patent blue2,5\% was injected for SLN detection Use 22G Spinal Needle 3 and 9 O'clock total 4 cc1cc superficial and 1cc deep on each side. 19 Mini Invasive Surgeries (MIS) and 42 Radical Histerectomy Type C (ARHT) were performed.

\section{RESULTS}

The patients in SLN+PL group (32/61) the average of lymph node dissects was 16 (8-25). The SLN had metastasis in $9.75 \%$ (3/32), two cases with H\&E staining and one case with ultrastaging/immunohistochemistry. Finally in the group with only SLN (9/61) the average of lymph node dissects was $2.6(2-4)$.

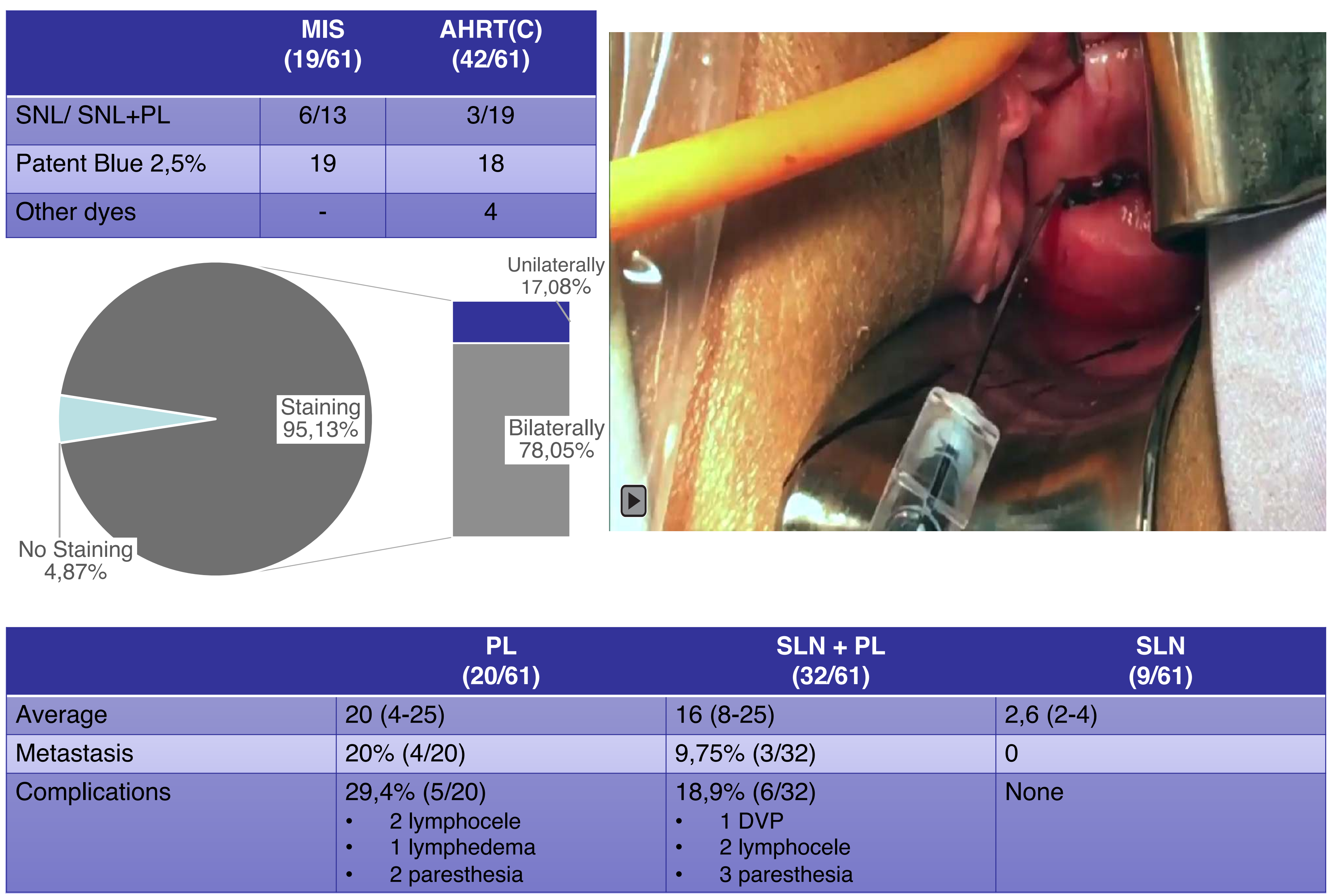

CONCLUSIONS: The SLN allows the detection of the first node in $78.05 \%$ bilaterally with $19.56 \%$ finding it in nonusual PL sites and with a low rate of complications related to PL. This technique is a valid alternative for centers that do not have the technological infrastructure to implement detection with indocyanine green. These results encourage the initiation of this modality in selected cases. To be able to advance in research lines that increase the detection of SNL. 